\title{
Comparison of Activity of the Muscles around the Shoulders during Push Up and Push Up Plus Exercises under Diverse Stabilization Conditions
}

\begin{abstract}
This study aimed to examine the most effective exercise method for shoulder joint rehabilitation by comparatively observing activity of shoulder stabilizer muscles during push up and push up plus exercises under diverse stable conditions.

The subjects were 20 healthy adults students who went to M university. While the subjects conducted push up and push up plus exercises under diverse stable conditions(a stable surface, a support of $25 \mathrm{~cm}$ height, a support of $30 \mathrm{~cm}$ height and a balance pad), activities of the upper trapezius muscle, pectoralis major muscle, serratus anterior muscle, and triceps brachii muscle were recorded.

During push up and push up plus exercises, activities of the stabilizer muscles were higher when the stable condition was changed rather than on the stable surface. In particular, when the support of $30 \mathrm{~cm}$ height and balance pads were applied, activity of the shoulder stabilizer was highest. There were significant differences in the upper trapezius muscle and triceps brachii muscle during the push up exercise(p<.05) and in the serratus anterior muscle during the push up plus exercise(p<.05).

Activities of the shoulder stabilizers were higher when the upper and lower limbs' surface stable conditions were changed than the stable surface. Therefore, when programs for rehabilitation of shoulder joints are applied, provision of diverse stable conditions according to patients' conditions will be effective methods.
\end{abstract}

Key words: Push-up plus; Surface conditions; Electrical stimulation

\author{
Han Ki Lee ${ }^{a}$, Jun Cheol Lee ${ }^{b}$ \\ Seong Min Yoon
}

aMasan University, Changwon; 'Samil Jungpool

Hospital, Changwon, Korea

Received : 09 November 2014

Revised : 10 Januaruy 2015

Accepted : 23 February 2015

Address for correspondence

Jun Cheol Lee, PT, MS.

Sam-il JungPoong Hospital, Changwon, Korea.

Tel: 82-01-3065-8511

E-mail : hklee1348@hanmail.net

* This research was financially supported by Masan University as part of 2014 industry academic research.

\section{INTRODUCTION}

In the modern society, abnormal movements of the scapula and humerus, in particular, resulting from biodynamic damages of the human body, are characterized by movement impairment syndrome of the scapula, which may trigger pain in certain areas and increase or decrease in range of motion. As a result, compensation exercise in certain directions and decrease in muscle strength appear (1).

The shoulder joints have the greatest movement range among all the joints of the human body(2) and are complex areas, and because of wide mobility, are fluid but affect stability and therefore are apt to trigger damages to the bones, muscles, tendons, ligaments, and bursas by excessive movements of the joint(3). Accordingly, the functions and stability of shoulder joints occurring by interaction and harmony among different joints are an important element in ordinary life(4).

Stabilization is an ability to adjust large or fine movements of the joints consciously or unconsciously and in dynamic stability of the shoulder joints the muscles play a great role, and normal movement pattern of the shoulder joints is harmonious movement together with stabilization rhythms of the scapular(3) and three dimensional movement, not two dimensional movement(5). The goal of stabilization exercise is to recover muscles and movement adjustment ability $(6,7)$.

The serratus anterior among many muscles which act in shoulder stabilization has been known 
as an important element to maintain normal scapulohumeral rhythm. The serratus anterior aids upward rotation and posterior tipping of the scapula while raising the upper limbs and prevents scapular winging by maintaining the scapula within the thoracic cage. A winged scapula is a condition where the internal side floats and the lower edge is protruded back(8) during the abduction of the arms and occurs due to damage to long thoracic nerve resulting from trauma around the scapula, a surgery, an infection, and damage related to sports activities(9).

When one comes down with this disease, the scapula noticeably floats when resistance is given at $30^{\circ}$ adduction or when $60^{\circ}$ abduction is conducted(3) and a general cause is the weakening of the serratus anterior and trapezius(9); This disease triggers loss of upper limb muscle strength and restriction to flexion and abduction, bringing about severe pain $(10,11)$. Therefore, the motions of holding, pulling, and pushing something are restricted and activities of daily living may be negatively affected(12).

Therapeutic exercises to recover shoulder joint functions are being carried out and in particular, the importance of coordination and balance adjustment among the stabilizer muscles composing the shoulder joints has been recognized(13). For such reason, shoulder rehabilitation program aimed at increasing strength of the serratus anterior and the stabilization muscles around the scapula and selection of an appropriate exercise in the process of implementing such program are important(14). In general, therapeutic exercise programs for prevention and rehabilitation of shoulder joint functional disability consider scapular stabilizer muscles importantly and recently, there is a lot of trend to utilize closed chain exercise in upper limb exercise and treatment programs(15).

Closed chain exercise includes movements when the peripheral areas of the body are fixed or stabilized at palm width and the representative example is weight supporting training. Closed chain exercise triggers $\mathrm{co}^{-}$-contraction of the multiple muscles through mechanical pressure on the articular surfaces as well as improving muscle strength and endurance. This exercise stimulates afferent nociceptors around the joints and provides more proprioceptors and therefore is often used in an exercise treatment program for dynamic stability of the joints and postural maintenance(17).
Push up is known as a typical upper limb closed chain exercise, an exercise for performance evaluation and improvement of muscle strength of the chest, shoulders, and arms, and a resistance exercise. This exercise is very popular in upper limb strengthening programs and easy to learn, does not require equipment, and may be applied differently from fitness level; Its popularity is growing (18). Recently, push up plus was created by adding the elements of plus to push up. A push-up plus is an exercise which added scapula traction to ordinary push up motion and Ghigiareli (18) recommended push up plus as an effective closed chain exercise for the serratus anterior muscle and scapular stabilizing synergist.

In a previous study, Moseley et al.(14) used push up plus and push up exercise on the area of the hands to conduct shoulder joint exercise and compared different muscles, in other words, upper, middle, and lower trapezius muscles, levator scapulae muscle, rhomboid muscle, middle and lower serratus anterior muscle, and pectoralis major muscle. Ludewig et al.(16) compared differences among four types of push up standard push up plus exercise, push up exercise modified on elbows(a modified push up with the elbows flexed), push up exercise modified on knee(a modified push up conducted while kneeling down), and push up exercise against a wall(a modified push up conducted while pushing the wall in a standing position) and examined activities of the serratus anterior and upper trapezius and rates of these two muscles.

In addition, Hwang(20) studied the effects of push-up plus exercise using a sling on stabilization of the lumbar region and $\mathrm{Oh}$ et al.(21) compared when instability was provided to the hands with when a stable surface was provided using sling exercise equipment. Lear and Gross(22) compared push up plus with push up plus conducted with the feet raised. Nonetheless, research which examined push up plus exercises according to the height of base of support, stable base of support, and unstable base of support is still insufficient.

Accordingly, this study intends to examine activities of shoulder stabilizer muscles according to diverse stabilization conditions during push up exercise and push up plus exercise used as closed chain exercise methods in upper limb exercise programs and look at the most effective exercise method for shoulder joint rehabilitation. 


\section{METHODS}

\section{Subjects}

This study involved 20 young male adults in the Department of Physical Therapy, M University who sufficiently listened to the purpose and method of this study and agreed to participate in this experiment. All the subjects had no experience on weight training and had no musculoskeletal system disease such as shoulder pain, winging scapula, impingement syndrome, and carpocarpal joint damage. The general characteristics of the subjects are as follows.

Table 1. General characteristics of the subjects

\begin{tabular}{cc}
\hline General Characteristics & Mean \pm SD \\
\hline Age & $24.05 \pm 2.21$ \\
Weight & $66.65 \pm 7.67$ \\
Height & $174.40 \pm 5.92$ \\
\hline
\end{tabular}

\section{Experimental tool}

In order to measure activities of the dominant pectoralis major muscle, serratus anterior muscle, upper trapezius muscle, and triceps brachii muscle, MP150 WSW(BIOPAC System Ine.CA, USA) (Fig. 1), a surface electromyogram was used.

Medical electrodes Ag/AgCI were used as electrodes. In order to provide an unstable surface, balance pads (Fig. 2) were applied to the bilateral palms and the supports of $25 \mathrm{~cm}$ height and $30 \mathrm{~cm}$ height were applied(Fig. 3, 4).

\section{Experimental method}

\section{Measurement by the Electromyogram}

In order to remove sweat, moisture, and electrical resistance, hair on the area where an electrode would be applied was removed and the skin was cleaned with alcohol gauze. The location of each muscle where the electrodes would be attached was determined by referring to the studies by Cram and Kneebone(23) and Lear and Gross(22). The dominant upper limb was determined by asking the subjects and the dominant upper limb of all the subjects was the right side.

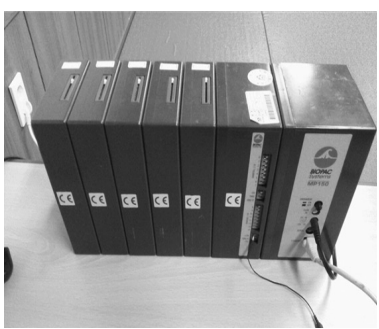

Fig. 1. Electromyogram

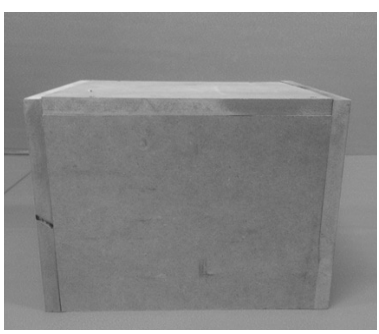

Fig. 3. Support of $25 \mathrm{~cm}$ height

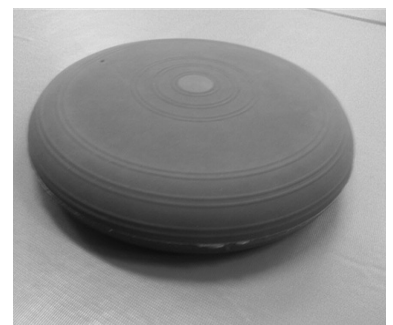

Fig. 2 Balance pad

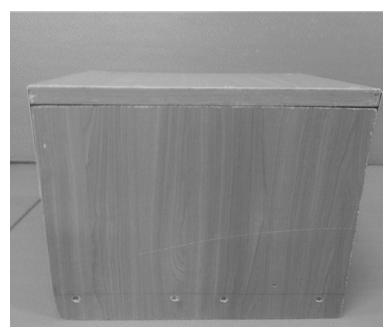

Fig. 4 Support of $30 \mathrm{~cm}$ height
Table 2 The location of the electrodes by each muscle

\begin{tabular}{cl}
\hline Measured muscle & \multicolumn{1}{c}{ Location of attachment } \\
\hline Pectoralis major & $\begin{array}{l}\text { The middle area between the } \\
\text { anterior axilla and fold in the } \\
\text { oblique direction from 2cm below } \\
\text { the clavicle }\end{array}$ \\
\hline Serratus anterior & $\begin{array}{l}\text { Anterior boundary of the latissimus } \\
\text { dorsi muscle, the height of lower } \\
\text { end of the scapula }\end{array}$ \\
\hline Upper trapezius & $\begin{array}{l}\text { The middle area between the } \\
\text { acromion of the scapula in the } \\
\text { direction in parallel with muscle } \\
\text { fibers and the 7th cervical verte- } \\
\text { bra's spinous process }\end{array}$ \\
\hline Triceps brachii & $\begin{array}{l}\text { The belly at the center of the back } \\
\text { of the upper arm }\end{array}$ \\
\hline
\end{tabular}

In order to quantify activity potential of each muscle, activity of each muscle during maximal voluntary isometric contraction(MVIC) in a manual muscle test position was measured. Data values for five seconds were processed into root mean squares(RMSs) and the average electromyography signal amount for three seconds excluding the first and last seconds was used as 100\% MVIC.

\section{Study procedure}

The starting posture of all exercises was to spread the hands shoulder width, gathered both feet, and maintained the body in a straight line (15). Push up 
exercise with the knee joints flexed needed less power than push up exercise with the knee joints extended while able to derive similar electromyogrphy amplitude(8). Accordingly, this study applied modified push up exercise with the knee joints flexed to the subjects.

In addition, in order to maintain a neutral posture of the cervical vertebrae, the craniocervical area was flexed so that the cervical vertabrae and the thoracic vertabrae were on a straight line(24). Before the experiment, all the subjects were asked not to excessively raise the thoracic vertabrae as a compensation action when they tracted their shoulders and practiced push up plus three times while maintaining their body in a straight line (25). In this study, the subjects performed the following exercises in a random order.

They performed the exercise with a basic posture (Fig. 5).

They performed the exercise with a support of $25 \mathrm{~cm}$ height being applied(Fig. 6).

They performed the exercise with a support of $30 \mathrm{~cm}$ height and balance pads on the bilateral hands being applied(Fig. 7).

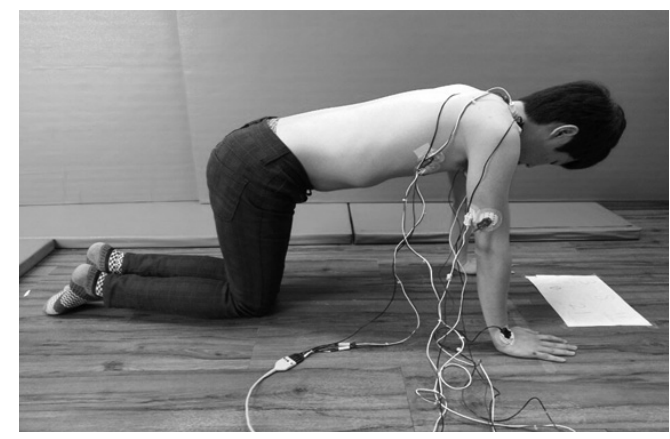

Fig. 5. Basic posture

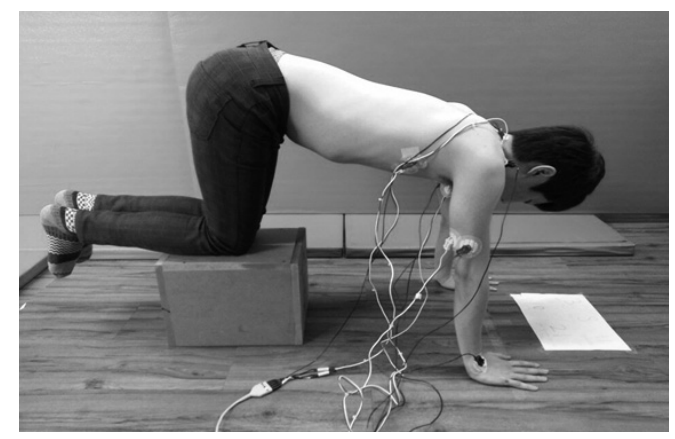

Fig. 6. Posture with a support of $25 \mathrm{~cm}$ height

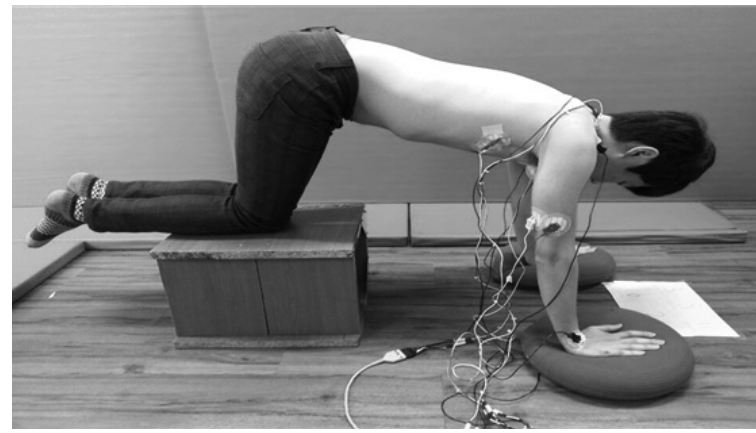

Fig. 7. Posture with a support of $30 \mathrm{~cm}$ height and balance pads

Under each measurement method, an oral instruction "start" was given. At a speed comfortable for the subjects, they conducted push-up and push-up plus motions. They took a rest for two minutes between each performance. Electromyography signals were collected while the subjects maintained scapular protraction with isometric contraction for five seconds and maintained isometric contraction in a push-up flexion motion for five seconds. Average electromyography signals for three seconds excluding the first and last seconds were used for analysis.

\section{Electromyography signal processing method}

Channels were designated so that each channel was able to receive electromyography signals. Surface electromyography signals and other signals were filtered and processed using Acknowledge 4.1 software on a personal computer. The sampling rate of the conducted signals was set at $1000 \mathrm{~Hz}$ and the amplified wave form was filtered using a band pass filter at $60 \sim 500 \mathrm{~Hz}$ and a notch filter at $60 \mathrm{~Hz}$. In order to quantify the collected signals, they were processed into RMS.

\section{RESULTS}

\section{Activity of the upper trapezius muscle according to different base of support}

During push up and push up plus exercises, activity of the upper trapezius muscle was higher in a posture with changes from the stable condition compared to on the stable surface. In particular, in a push up exercise posture, there were statistically significant differences according to each condition of the supports(p<.05). However, there were no significant differences in a push up plus exercise posture(Table 3). 
Comparison of Activity of the Muscles around the Shoulders during Push up and Push up Plus Exercises under Diverse Stabilization Conditions

Table 3. Activity of the upper trapezius muscle according to the different bases of support

\begin{tabular}{clc}
\hline Posture & \multicolumn{1}{c}{ Base of support condition } & Mean \pm SD \\
\hline \multirow{2}{*}{ Push up } & Stable surface & $42.35 \pm 24.43$ \\
& Support of $25 \mathrm{~cm}$ height & $64.17 \pm 43.16$ \\
& Support of $30 \mathrm{~cm}$ height and balance pads & $70.84 \pm 45.12$ \\
\hline \multirow{3}{*}{ Push up plus } & Stable surface & $14.46 \pm 11.15$ \\
& Support of $25 \mathrm{~cm}$ height & $18.06 \pm 14.22$ \\
& Support of $30 \mathrm{~cm}$ height and balance pads & $19.24 \pm 15.50$ \\
\hline
\end{tabular}

Activity of the pectoralis major muscle according to the different bases of support

During push up and push up plus exercises, activity of the pectoralis major muscle was higher in a posture with changes from the stable condition compared to on the stable surface. However, there were no statistically significant differences among the three methods(Table 4).

Table 4. Activity of the pectoralis major muscle according to the different bases of support

\begin{tabular}{|c|c|c|c|}
\hline Posture & Base of support condition & Mean $\pm S D$ & $\mathrm{p}$ \\
\hline \multirow{3}{*}{ Push up } & Stable surface & $27.12 \pm 13.73$ & \multirow{3}{*}{.278} \\
\hline & Support of $25 \mathrm{~cm}$ height & $30.16 \pm 13.93$ & \\
\hline & Support of $30 \mathrm{~cm}$ height and balance pads & $39.67 \pm 39.40$ & \\
\hline \multirow{3}{*}{ Push up plus } & Stable surface & $10.10 \pm 6.41$ & \multirow{3}{*}{.140} \\
\hline & Support of $25 \mathrm{~cm}$ height & $7.61 \pm 5.13$ & \\
\hline & Support of $30 \mathrm{~cm}$ height and balance pads & $12.99 \pm 8.63$ & \\
\hline
\end{tabular}

\section{Activity of the serratus anterior muscle according} to the different bases of support

During push up and push up plus exercises, activity of the serratus anterior muscle was higher in a posture with changes from the stable condition compared to on the stable surface. In partic- ular, in a push up plus exercise posture, there were statistically significant differences according to each of the supports(p<.05). However, there were no significant differences in a push up exercise posture(Table 5).

Table 5. Activity of the serratus anterior muscle according to the different bases of support

\begin{tabular}{clll}
\hline Posture & \multicolumn{1}{c}{ Base of support condition } & Mean \pm SD & p \\
\hline \multirow{3}{*}{ Push up } & Stable surface & $29.58 \pm 20.67$ & \\
& Support of $25 \mathrm{~cm}$ height & $43.21 \pm 16.26$ & .053 \\
& Support of 30cm height and balance pads & $39.71 \pm 20.71$ & \\
\hline \multirow{3}{*}{ Push up plus } & Stable surface & $42.76 \pm 18.06$ & .000 \\
& Support of $25 \mathrm{~cm}$ height & $62.92 \pm 20.02$ & $82.87 \pm 21.75$ \\
& Support of 30cm height and balance pads & 8
\end{tabular}




\section{Activity of the triceps brachii muscle according to the different bases of support}

During push up and push up plus exercises, activity of the triceps brachii muscle was higher in a posture with changes from the stable condition compared to on the stable surface. In particular, in a push-up exercise posture, there were statistically significant differences according to the condition of each support(p<.05). However, there were no significant differences in a push-up plus exercise posture(Table 6).

Table 6. Activity of the triceps brachii muscle according to the different bases of support

\begin{tabular}{clll}
\hline Posture & \multicolumn{1}{c}{ Base of support condition } & Mean \pm SD & $p$ \\
\hline \multirow{3}{*}{ Push-up } & Stable surface & $65.53 \pm 17.39$ & \\
& Support of $25 \mathrm{~cm}$ height & $80.27 \pm 15.68$ & .000 \\
& Support of 30cm height and balance pads & $78.64 \pm 16.33$ & \\
\hline \multirow{3}{*}{ Push-up plus } & Stable surface & $53.85 \pm 25.74$ & .052 \\
& Support of $25 \mathrm{~cm}$ height & $60.65 \pm 25.81$ & \\
& Support of 30cm height and balance pads & $61.04 \pm 26.02$ & \\
\hline
\end{tabular}

\section{DISCUSSION}

Push up plus exercise is considered as the most appropriate exercise to heighten activities of the muscles around the scapula including the serratus anterior muscle and therefore has been most preferred $(8,14,16,22)$. It is an exercise to most actively move the serratus anterior muscle on the electromyography and under the condition of maintaining the posture, conducting push-up maintains maximal contraction of the serratus anterior muscle during the movement of the elbow joints, thereby reducing protruded scapula and increasing ability of the scapula's upward rotation. As an effective method for this, a modified pushup posture in a quadruped position is recommended(26) and conducting exercise with the activated condition of the serratus anterior muscle maintained is more effective for scapula treatment (24, 27).

Oh et al.(21) noted that activity of the pectoralis major muscle was significantly higher in push up motion using a sling than on a fixed base of support and in the case of triceps brachii muscle, there was no difference between the sling and the fixed base of support but muscle activity was high when the elbow joint angle was 45 degrees. Park et al.(28) observed that during push up exercise on a stable and an unstable base of support, activity of the serratus anterior muscle became significantly higher but there was no difference between the activity of the upper trapezius muscle and that of the lower trapezius muscle.

Lehman et al.(29) and Kim(27) reported that when push up plus exercise was conducted on a stable and an unstable base of support, activity of the serratus anterior muscle significantly differed according to the change in foot height regardless of the condition of the surface where the hands contacted. In a study by Kim and Lee(30), when an unstable base of support was provided to the lower extremities, muscle activity more obviously increased even though it did not statistically significantly improve, than when a stable and un unstable surface were provided to the upper extremities. In addition, in studies by Lear and Gross(21) and Ludewig et al.(15), which examined correlation between the serratus anterior muscle and the upper trapezius muscle, when the subjects conducted push up plus with the bilateral feet on a small chair, there was inverse correlation between the serratus anterior muscle and the upper trapezius muscle and the lower trapezius muscle did not show significant muscle activity.

Accordingly, this study examined muscle activity of the shoulder stabilizer muscles according to different surface condition in a push up plus position used as a method of closed chain exercise in an upper extremity exercise program and intended to provide the most effective exercise method for shoulder joint rehabilitation.

Therefore, in the present study as well like previous studies, push up plus exercise was conducted in a quadruped position, and changes in the distance 
between the two hands affected muscle activity of the applied muscles(31). Accordingly, in order not to make errors, this study applied the distance between the two hands constantly to be the shoulder width of the subjects. In addition, special attention was paid so that the body was not under the shoulders in order to prevent semiluxation of the humerus and not to damage the joint capsule of the shoulder joints' ligaments(30).

The result was that activity of the pectoralis major muscle and the serratus anterior muscle did not significantly differ in a flexion position according to different bases of support and there was significant difference between the upper trapezius muscle and the triceps brachii muscle. There was no significant difference in activities of the upper trapezius muscle and the pectoralis major muscle in push up plus position according to different bases of support but there was significant difference only in activity of the serratus anterior muscle. In previous research, push up plus exercise conducted in a quadruped position exhibited the highest activation of the serratus anterior muscle $(8,26)$ but did not show activation of the upper trapezius muscle. The reason is that the subjects who participated in this study were healthy males in their 20s and had no imbalance between the upper trapezius muscle and the serratus anterior muscle.

According to previous research, patients with imbalance between the trapezius and the serratus anterior exhibit excessive muscle activity in order to compensate for the serratus anterior muscle with the weakened upper trapezius muscle and as a result abnormal rotation of the scapula occurs triggering impingement(15, 25). An exercise with low ratio of the trapezius to the serratus anterior is one of important elements for rehabilitation exercise aimed at selectively strengthening the serratus anterior muscle, reducing muscle imbalance, and stabilizing the shoulder(16).

Functional disability of the shoulders is a musculoskeletal disease which occurs during ordinary life and some of ordinary injuries are shown in instability of the shoulders and impingement syndrome. Existing research noted that in order to resolve imbalance of the muscles around the scapula and control wrong nerve roots, selective strengthening exercise according to weakened muscles and adequate exercise stimulation are required(6).

The limitation of this study is that precise effect cannot be concluded because it did not involve patients with functional disability of the shoulders and it cannot be generalized to all the people because it concerned only 20 males in their 20 s. Therefore, future research should examine an exercise program to increase the number of subjects including patients with functional disability of the shoulders and to improve all the surrounding muscles.

\section{CONCLUSION}

This study examined activity of the shoulder stabilizer muscles in a push up exercise posture and a push up plus exercise posture according to diverse surface stabilization conditions and intended to provide the most effective exercise method for shoulder joint rehabilitation. This study applied a stable surface, a support of $25 \mathrm{~cm}$ height, a support of $30 \mathrm{~cm}$ height, and balance pads and compared activities of the upper trapezius, pectoralis major muscle, and triceps brachii muscle.

The serratus anterior muscle important for the protruded scapula exhibited the highest muscle activity in an unstable push up plus exercise and higher muscle activity when a support of $25 \mathrm{~cm}$ height, a support of $30 \mathrm{~cm}$ height, and a balance pad were applied than when a stable surface was applied.

Therefore, provision of diverse bases of support according to the conditions of patients in a program for shoulder joint rehabilitation will a more effective method than a sable base of support.

In addition, according to the purpose of this study, applying effective base of support conditions and postures for each muscle for the rehabilitation of the shoulder joints will be an effective method.

\section{Acknowledgement}

This research was financially supported by Masan University as part of 2014 industry academic research. 


\section{REFERENCES}

1. No TH, Kim JH, Park SB, Lee NN. Analysis of biomechanics of push up movement. J. of the Korean Society of Physical Medicine. 2009; 4(4): 269-274.

2. Warby SA, Pizzari T, Ford JJ, Hahne A. J, Watson L. The effect of exercise based management for multidirectional instability of the glenohumeral joint: a systematic review. Journal of Shoulder and Elbow Surgery. 2014; 23(1): 128142 .

3. Magee DJ. Orthopedic Physical Assessment. St. Louis, Missouri : Elsevier. 2014.

4. Hess SA. Functional stability of the glenohumeral joint. Man Ther. 2000; 5(2): 63-71.

5. Bae SS, Choi JW, Ching HA et al. Biomechanical analysis of scapular pattern in proprioceptive neuromuscular facilitation. J Kor Soc Phys Ther. 1999; 11(3): 65-69.

6. Anderson GS, Gaetz M, Holzmann M, Twist P. Comparison of EMG activity during stable and unstable push up protocols comparison of EMG activity during stable and unstable push up protocols. European J of Sport Science. 2013; 13(1): $42-48$.

7. Lee S. The effect of hand position changes on electromyographic activity of shoulder stabilizers during push up plus exercise on stable and unstable surfaces. Journal of Physical Therapy. 2013; 25(8): 981-984.

8. Decker MJ, Hintermeister RA, Faber KJ, Hawkins RJ. Serratus anterior muscle activity during selected rehabilitation exercises. Am J Sports Med, 1999; 27(6): 784-791.

9. Fiddian NJ, King RJ. The winged scapula. Clin Orthop Relat Res. 1984; 185: 228-236.

10. Yoon JG. Periscapular muscle activities and kinematic analysis of the performed on different supporting surfaces for the lower limbs push-up plus exercise. Journal of Physical Therapy Science. 2013; 25(3): 259-262.

11. Snarr R, Esco MR. Exercise technique push up with knee tuck using a suspension device. Stretch and Conditioning Jouranl. 2013; 35(2): 30-32.

12. Kim JS, Lee DY. A effect of the shoulder stabilizer muscle activity during a push up plus on a different condition surface. The Journal of digital policy \& management. 2012; 10(1): 399-405.

13. Lehman GJ, Gilas D, Patel U. An unstable support surface does not increase scapulothoracic stabilizing muscle activity during push up and push up plus exercises. Manual Therapy. 2008; 13(6): 500-506.

14. Moseley JB Jr, Jobe FW, Pink M, Perry J, Tibone J. EMG analysis of the scapular muscles during a shoulder rehabilitation program. Am J Sports Med. 1992; 20(2): 128-134.

15. Ludewig PM, Cook TM. Alterations in shoulder kinematics and associated muscle activity in people with symptoms of shoulder impingement. Phys Ther, 2000; 80(3): 276-291.

16. Ludewig PM, Hoff MS, Osowski EE, Meschke SA, Rundguist PJ. Relative balance of serratus anterior and upper trapezius muscle activity during push up exercises. Am J Sports Med. 2004; 32(2): 484-493.

17. Ellenbecker TS, Davies GJ. Closed Kinematic Chain Exercise: A comprehensice guide to multiple joint exercise. Champaign, IL. Human Kinetics Publishers, 2001; 53-58.

18. Marina K. Gouvall and Konstantinos boudolos, Dynamic and electromyogaphical analysis in variants of push up exercise. Journal of Strength and Conditioning Research, 2005; 19(1): 145-151.

19. Ghigiarelli, JJ. Exercise technique advanced loading variations in the push up exercise. Stretch and Conditionag Journal. 2013; 35(4): 107-110.

20. Hwang, HJ. The Effects of Push-up Plus Exercise using a Sling on Lumbar Region Stabilization. Master's Thesis, Korea Graduate School. 2011

21. Oh JS, Park JS, Kim SY, Kwon OY. Comparison of muscle activity during a push up on a suspension sling and a fixed support. Physical Therapy Korea. 2003; 10(3): 29-40.

22. Lear LJ, Gross MT. An electromyographical analysis of the scapular stabilizing synergists during a push up progression. J Orthop Sports Phys Ther, 1998; 28(3): 146-157.

23. Cram JR, Kneebone WJ. Cervical flexion: A study of dynamic surface flectromyography and range of motion. Journal of manipulative and physiological therapeutics. 1999; 22(9): 570-575.

24. Jeon YJ, Heong YJ, Kim SH, Shin HS. Selective activation of serratus anterior using electromyography biofeedback during push up plus. Physical Therapy Korea. 2011; 18(1): 1-8.

25. Park JS, Jeon HS, Kwon OY. A comparison of the shoulder stabilizer muscle activities during push up plus between persons with and without winging scapular. Physical Therapy Korea. 2007; 14(2): 44-52.

26. Kim SH, Kwon OY, Kim SJ, Park KN. Serratus anterior muscle activation during knee push up 
plus exercise performed on static stable, static unstable, and oscillating unstable surfaces in healthy subjects. Physical Therapy in Sport, 2014; 15(1): 20-25.

27. Kim MH. Effects of push-up exercise with hip adduction on the COP deviation and the serratus anterior and L1 paraspinal muscles. J. of Physical Therapy Science. 2013; 25(7): 783-784.

28. Park SK, Lee HO, Kim JS, Kim SY. A comparison of muscle activity in periscapular muscles during push-up plus exercise on stable support and unstable support. Journal of Korean Academy of Orthopaedic Manual Therapy. 2005; 11(2): 71-82.

29. Lehman GJ, Gilas D, Patel U. An unstable support surface does not increase scapulothoracic stabilizing muscle activity during push-up and pushup plus exercises. Man The, 2008; 13(6): 500506.

30. Kim JS, Lee DY. A effect of the shoulder stabilizer muscle activity during a push-up-plus on a different condition surface. The Korea Society of Digital Policy \& Management. 2012; 1091: 399405.

31. Starnberger I. Kamminga P. Fosah VC. Nuttma C. The Push-Up' as a calling posture in nectophrynoides tornieri(Anura: Bufonidae) in the amani nature reserve, Tanzania. Herpetologia 2011; 67(2): 124-134. 